\title{
Combining Nanoprobes and the Electron Microscope - Challenges and Opportunities
}

\author{
L.-M. Peng ${ }^{1,2}$, Q. Chen ${ }^{1}$, J.Y. Wang ${ }^{1}$, M.S. Wang ${ }^{1}$, C.H. Jin ${ }^{2}$ and S. Wang ${ }^{1}$ \\ ${ }^{1}$ Key Laboratory for the Physics and Chemistry of Nanodevices and Department of Electronics, \\ Peking University, Beijing 100871, China \\ ${ }^{2}$ Beijing Laboratory of Electron Microscopy, Institute of Physics, Chinese Academy of Sciences, \\ P.O. Box 603, Beijing 100080, China
}

While electron microscopes have been used for decades for high resolution characterization and sometimes also for property measurements, e.g. electronic structure via EELS, optical excitations via $\mathrm{CL}$, most of the time the role played by the electron microscopist is more passive than active. The development of nanoscale science and technology demands a more active role by the microscopists and property measurement of nanoscale functional units is one of the main challenges. To perform such a measurement one has to have a mean of accessing the nanostructure with high precision while not damaging it or introducing two much perturbation to the system. Conventionally in microelectronics such an access to the IC units is provided by microprobes. The movement of the probe is monitored by optical microscope, and that limits the ultimate precision over which the probe may be controlled. On the other hand the scanning probe microscope (SPM) offers extremely high level of control on the movement of its probe. But at the same time the view of the SPM is severely restricted by the high level control and usually only atomic smooth objects were explored using SPM.

Nanoscale probes are being introduced into the electron microscopes and providing vast amount of spatially resolved information on, to name a few, the local electronic structure, mechanical and transport properties of the nanostructures. In our laboratory at Peking University a miniaturized SPM has been installed in a single tilt TEM sample stage and four MM3A nanoprobes have been installed in a SEM equipped with a Gatan cold stage. Preliminary experiments [1-4] using these instruments result in valuable information concerning the transport (Fig. 1) and electron field emission properties (Fig. 2) of single nanostructures. In-situ electron field-emission experiments carried out inside the TEM revealed that the field-emission parameters, e.g. the threshold voltage and the field conversion factor, depend in general both on the tip-CNT distance and on the shape of both the tip and the CNTs. As a result comparisons between measured parameters for different materials should be made extremely carefully and under at least similar if not identical experimental set up.

The nanoprobe system in the electron microscope may also be used effectively for setting up electric contacts to nanostructures and to perform transport measurement, to manipulate and fabricate nanoobjects, e.g. nanocavity for nanolasing applications, to explore the electromechanical response of the NEMS system and to study the wedding behaviour at nanoscale. The implication of combining the probe system with conventional electron microscopic function is far reaching, and we expect to see more developments and breakthroughs in the coming years as the development of nanoscale science and technology demands more and more on the fundamental understanding as well as the ultimate control on the nanoobject with atomic precision.

\section{References}

[1] L.-M. Peng et al., MICRON 35 (2004) 495

[2] Q. Chen and L.-M. Peng, Appl. Phys. Lett. 84(24) (2004) 4920

[3] M.S. Wang et al., Materials Science Forum 475-479 (2005) 4071

[4] M.S. Wang, L.-M. Peng, J.Y. Wang and Q. Chen, J. Phys. Chem. B 109(1) (2005) 110 
[5] This work was supported by the National Science Foundation of China (Grant Nos 10434010 and 90206021), the Chinese Ministry of Education (Key Project, Grant No 10401) and Peking University.
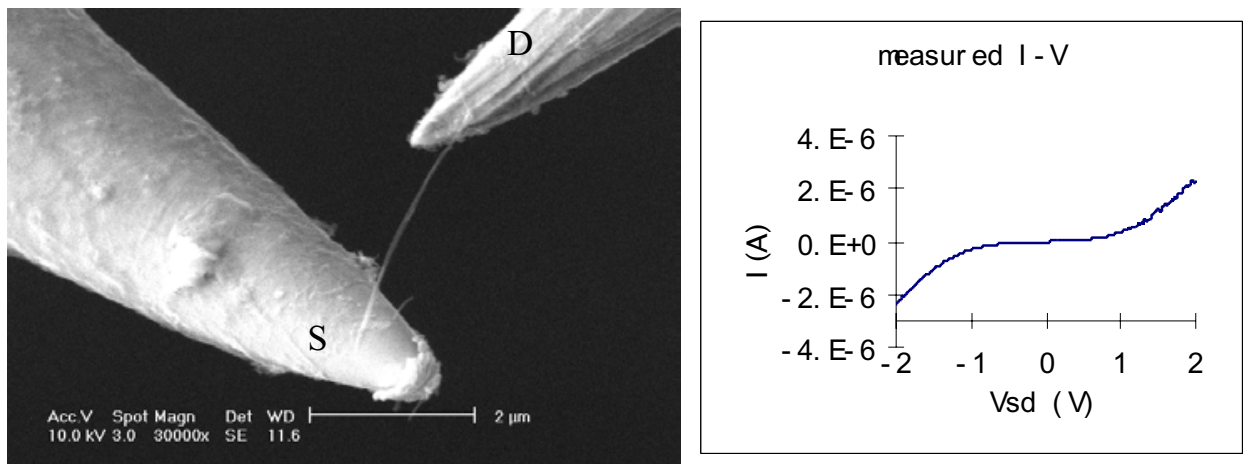

Fig. 1. A two-probe set up and results for a two-terminal measurement on the electron transport in a carbon nanotube.

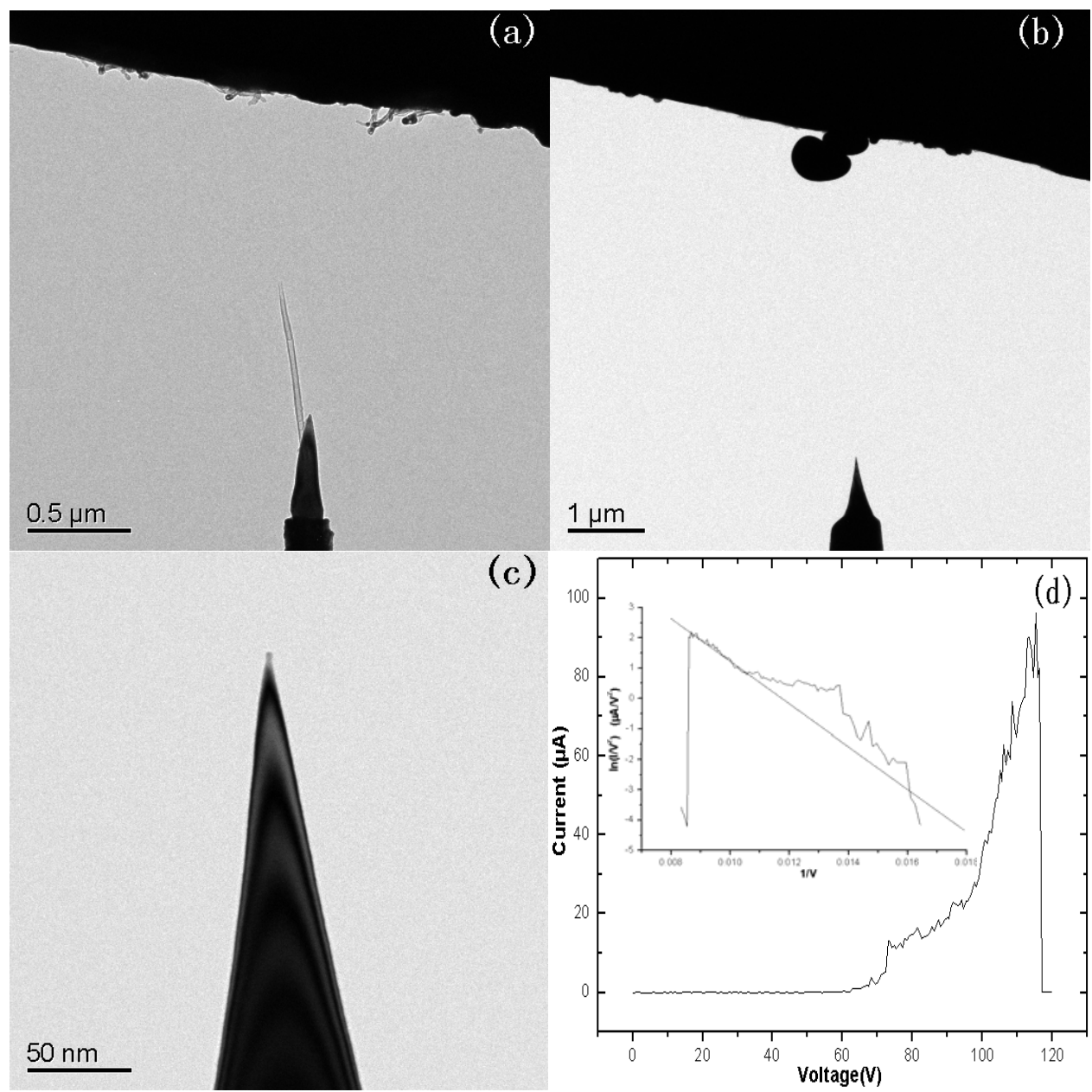

Fig. 2. TEM images showing (a) the failure of the electron field-emission of a carbon nanotube occurs at $\sim 100 \mu \mathrm{A}$, and at the CNT/W contact point via local heating and field evaporation. Some metal $\mathrm{W}$ atoms were seen to have been transferred to the counter Pt electrode (b), and an extremely sharp Wtip $(\sim 2 \mathrm{~nm})$ was formed (c). 